\title{
End-of-life care for patients with COPD in the community setting
}

Chronic obstructive pulmonary disease (COPD) is a common chronic disease which causes significant mortality and morbidity. The chronic nature of the disease results in patients and carers generally living with the illness for longer than patients with a cancer diagnosis. In the earlier stages of the disease, management focuses on improving symptoms and exercise capacity and reducing exacerbations.

As the disease progresses, a palliative care approach to symptom control and the adoption of strategies which accept dying as a normal process is appropriate. This should integrate the psychological and spiritual aspects of patient care and offer a support system to help patients live as actively as possible until death. ${ }^{1}$

Altering the paradigm to holistic palliative care as death approaches ${ }^{2}$ is supported in national guidance from bodies such as $\mathrm{NICE},{ }^{3}$ but defining when this should take place is difficult and in practice it is usually a gradual transition. ${ }^{4}$ As a large part of endof-life care for patients with COPD occurs in the community, it falls to the primary healthcare team with the support of specialist palliative care and secondary care services to provide this integrated care.

The best available data suggest there are approximately 900000 patients diagnosed with COPD in England and Wales, ${ }^{5}$ and allowing for known levels of underdiagnosis, the true number is likely to be around 2 million. ${ }^{3,5}$ Over 25000 people died of COPD in the UK last year, ${ }^{6}$ representing around $5 \%$ of all deaths, but this is likely to be an underestimate. ${ }^{7}$

The challenge for primary care physicians and researchers is making sense of the epidemiology at a practice and individual level in terms of identifying the population with $\mathrm{COPD}$, gauging when a palliative care approach is appropriate, and coordinating services for patients and carers.

At individual practice level, the identification of patients with COPD has been refined through the requirements of the Quality and Outcomes Framework
$(\mathrm{QOF})^{8}$ and greatly facilitated by the increased availability of spirometry, ${ }^{9}$ although there still appear to be deficiencies in the quality and interpretation of spirometry performed in primary care. ${ }^{10}$

The QOF is an annual reward and incentive programme for GPs, which was introduced as part of the new contract for GPs in 2004. It awards achievement points for meeting specific quality targets in the management of some common chronic diseases, including COPD. In 2006 points for palliative care were introduced. GPs are paid on the basis of the number of points achieved, but the QOF's impact on improving the quality of care remains uncertain. ${ }^{11}$

The introduction of palliative care registers as part of the QOF may aid in the identification of patients thought to be in the end stage of their disease, but this has yet to be proven. A GP is likely to have spent considerable time over a number of years in face-to-face contact with patients with COPD, ${ }^{12}$ and increasingly more time as death approaches. ${ }^{13}$ Patients with COPD appreciate continuity of care and reassurance provided by the primary healthcare team ${ }^{14,15}$ and the GP is in a key position to deliver and coordinate end-oflife care for patients with COPD.

Identifying the final phase of life is not a precise science, but the following features relate to a poor prognosis in COPD: severe airflow obstruction (that is, FEV1 less than $30 \%$ predicted value); frequent exacerbations and hospital admissions; requirement for long-term oxygen treatment; and development of cor pulmonale., ${ }^{3,16}$ The clinician's expectation of patient's death within the next 12 months has recently been proposed as being a strong indication for adopting a palliative care approach, ${ }^{17}$ but this may be difficult in COPD and an expectation of death in the next 6 months might be more realistic. Although research is required to determine the accuracy of the prognostic assessment, the clinician's attempt to define the final phase of life serves as an alert to possible palliative care needs.

Patients with severe COPD need to be given honest and clear information. A study specifically examining their needs identified five topics which seem concordant with our experience: diagnosis and disease process; treatment; prognosis; what dying might be like; and advance care planning. ${ }^{18}$ The barriers experienced by patients and clinicians to discussing end-of-life care differ, ${ }^{19}$ and help to explain difficulties around this sensitive area.

A New Zealand study of GP discussion of prognosis in COPD yielded practical strategies for use in general practice.$^{20}$ These were: be aware of implications of diagnosis; use uncertainty to ease discussion; build relationship with patients; be caring and respectful; begin discussion early in disease course; identify and use opportunities to discuss prognosis; and work as a team. Patients with advanced disease are often receptive to the dual agenda: 'Hope for and expect the best and prepare for the worst'. ${ }^{21}$

Advance care planning is seen by some as a way of preparing for the worst. This approach has been implemented in the US and has been shown to improve patients' satisfaction with their care as well as allowing them to make decisions about foregoing life-saving treatment and hospitalisation which remain unchanged even when near death. ${ }^{22}$ However, how such initiatives might transfer to primary care settings in other countries is uncertain.

A majority of UK GPs acknowledged a need to discuss prognosis in severe COPD, but this was not reflected in their reported behaviour. ${ }^{23}$ This may in part be explained by the phenomenon of 'response shift': the process whereby in the face of severe disease or impending death, individuals abandon their usual 'roadmap' of values and adopt new perspectives. ${ }^{24}$ Although this has not been specifically demonstrated for COPD, there is ample evidence that clinicians caring for patients with cancer consistently 
overestimate prognosis. ${ }^{25} \mathrm{~A}$ helpful development is a move from a prescriptive advance directive to an approach which involves discerning the patient's core values and acting on them while understanding that there may be changes in some areas, such as place of death. ${ }^{26}$

\section{SYMPTOMS}

Many studies confirm the predominance of the symptoms of breathlessness, anorexia, and constipation in severe COPD,,$^{14,27-30}$ along with significant problems with fatigue, pain, anxiety and depression, and poor sleep. The evidence base for symptom control in end-stage COPD is poor and management relies on a mix of evidence, considered best practice and experience. ${ }^{31}$

The problem of refractory dyspnoea is common and hard to treat. Optimised bronchodilator therapy, oxygen, and opioids remain the main stays of palliative pharmacological treatment. Clinicians should be aware of the dangers of respiratory depression with opioids, but once a palliative approach has been adopted symptom control should take precedence. Ideally, the rationale for this approach should be discussed with patients and their families.

A systematic review ${ }^{32}$ supported the continued use of oral and parenteral opioids to treat dyspnoea in advanced disease, and more specific data regarding use of oral opioids have been published. ${ }^{33}$ The usefulness of nebulised opioids is unclear, but it appears that they are no better than nebulised normal saline. In patients with end-stage disease oxygen is used to provide symptomatic relief of dyspnoea. Patients report considerable symptomatic benefit with short-burst oxygen, although there is little evidence for the effectiveness of oxygen in the palliation of breathlessness. The significant reported levels of pain imply under-treatment and could be linked to under prescription of opioid drugs due to fear of respiratory depression.

Weight loss and associated muscle wasting is a common feature in patients with severe COPD. The pathophysiology is incompletely understood and interventions with calorie and protein supplements produce only limited success. Patients should be encouraged to snack on high energy foods, taking small amounts frequently throughout the day. Anxiety and depression and insomnia are commonly associated with COPD, especially in patients with hypoxia and severe dyspnoea. NICE guidance on $\mathrm{COPD}^{3}$ recommends that patients who are found to be depressed or anxious should be treated with conventional pharmacotherapy and this should be accompanied by an explanation as to why depression needs to be treated alongside the physical disorder. There is, however, still a need to improve the evidence base on the most appropriate and effective methods to manage these systemic aspects of COPD.

\section{SECONDARY AND SPECIALIST PALLIATIVE CARE}

For some patients with COPD admission to hospital with an exacerbation and respiratory failure is the first contact with secondary care, and this may make it difficult for clinicians to form a balanced opinion about whether active or palliative care approaches are appropriate. However, some patients with severe COPD initially receive secondary care input in outpatient clinics and perhaps from respiratory nurse specialists. Such nurses have been shown to increase satisfaction with care and improve patient education, but these are as yet of unproven benefit in patients with severe disease and those who are dying. ${ }^{34}$

It appears that some patients and carers benefit from the reassurance and the surveillance that secondary care services can provide. ${ }^{14}$ Who benefits from secondary care, and why, deserves further clarification. There will come a point for many patients when community-based care with a predominant palliative element is more appropriate. Judging this transition point requires experience, sensitivity, and open communication among the hospital team, GP, and patient. Once this transition point is reached, it is crucial that the patient is not lost to follow-up and that planning of longterm support occurs. New initiatives in community palliative care, such as the UK Gold Standards Framework ${ }^{35}$ and palliative care registers, may be of benefit in planning such care, but evidence is as yet lacking.

Most specialist palliative care services in the UK accept patients with non-malignant life-threatening illness. This openness should increase with the publication of NICE guidelines, which encourage a palliative care approach for patients with severe COPD. ${ }^{3}$ The issue of referral needs to be discussed sensitively with patients with an emphasis on the positive aspects of specialist palliative care input such as help with symptom control, psychosocial support, as an information resource and bereavement follow-up.

\section{David MG Halpin,}

Consultant Respiratory Physician and Honorary Senior Clinical Lecturer, Royal Devon and Exeter Hospital Wonford, Exeter

\section{Clare J Seamark,}

GP, Honiton Research Practice, Honiton Devon

\section{David A Seamark,}

Lead Research GP, Honiton Research Practice, Honorary Senior Clinical Lecturer, Peninsula Medical School, Honiton Devon

\section{REFERENCES}

1. World Health Organization. WHO definition of palliative care.

http://www.who.int/cancer/palliative/definition/en/ (accessed 30 Apr 2008).

2. Lehman R. How long can I go on like this? Dying from cardiorespiratory disease. Br J Gen Pract 2004; 54(809): 892-893.

3. National Collaborating Centre for Chronic Conditions. Chronic obstructive pulmonary disease. National clinical guideline on management of chronic obstructive pulmonary disease in adults in primary and secondary care. Thorax 2004; 59(Suppl 1): 1-232.

4. Back AL, Curtis JR. When does primary care turn into palliative care? West J Med 2001; 175(3): 150-151.

5. Healthcare Commission. Clearing the air. A national study of chronic obstructive pulmonary disease. London: Commission for Healthcare Audit and Inspection, 2006.

6. National Statistics. Health statistics quarterly 34: web supplement. London: The Stationery Office, 2007.

7. Mannino DM, Brown C, Giovino GA. Obstructive lung disease deaths in the United States from 1979 through 1993. An analysis using multiple-cause mortality data. Am J Respir Crit Care Med. 1997; 156(3 Pt 1): 814-818.

8. Health and Social Care Information Centre. National Quality and Outcomes Framework Statistics for England 2004/05. Statistical Bulletin 2005/02/HSCIC. London: Health and Social Care Information Centre, 2005.

9. Halpin DM, O'Reilly JF, Connellan S, et al. Confidence and understanding among general practitioners and practice nurses in the UK about diagnosis and management of COPD. Respir Med 2007; 101(11): 2378-2385.

10. White P, Wong W, Fleming T, Gray B. Primary care spirometry: test quality and the feasibility and usefulness of specialist reporting. Br J Gen Pract 2007; 57(542): 701-705.

11. Roland M. The Quality and Outcomes Framework: too early for a final verdict. Br J Gen Pract 2007; 57(540): 525-527.

12. Gray DP. Forty-seven minutes a year for the patient. $B r$ J Gen Pract 1998; 48(437): 1816-1817.

13. Seamark C, Seamark D. Life limiting illness: the illness experience. In: Mitchell G (ed.). Palliative care: $a$ patient-centred approach. Oxford: Radcliffe Publishing, 2008. 
14. Seamark DA, Blake SD, Seamark CJ, Halpin DM. Living with severe chronic obstructive pulmonary disease (COPD): perceptions of patients and their carers. An interpretative phenomenological analysis. Palliat Med 2004; 18(7): 619-625.

15. Exley C, Field D, Jones L, Stokes T. Palliative care in the community for cancer and end-stage cardiorespiratory disease: the views of patients, lay-carers and health care professionals. Palliat Med 2005; 19(1): 76-83.

16. Halpin DM. Assessing the severity of COPD. Prim Care Respir J 2006; 15(2): 78-80.

17. Murray SA, Boyd K, Sheikh A. Palliative care in chronic illness. BMJ 2005; 330(7492): 611-612.

18. Curtis JR, Wenrich MD, Carline JD, et al. Patients' perspectives on physician skill in end-of-life care: differences between patients with COPD, cancer, and AIDS. Chest 2002; 122(1): 356-362.

19. Knauft E, Nielsen EL, Engelberg RA, et al. Barriers and facilitators to end-of-life care communication for patients with COPD. Chest 2005; 127: 2188-2196.

20. Halliwell J, Mulcahy P, Buetow S, et al. GP discussion of prognosis with patients with severe chronic obstructive pulmonary disease: a qualitative study. $\mathrm{Br} \mathrm{J}$ Gen Pract 2004; 54(509): 904-908.

21. Hansen-Flaschen J. Chronic obstructive pulmonary disease: the last year of life. Respir Care 2004; 49(1): 90-97.

22. Tierney WM, Dexter PR, Gramelspacher GP, et al. The effect of discussions about advance directives on patients' satisfaction with primary care. J Gen Intern Med 2001; 16(1):32-40.

23. Elkington $H$, White P, Higgs R, Pettinari CJ. GPs' views of discussions of prognosis in severe COPD. Fam Pract 2001; 18(4): 440-444.

24. Schwartz C. Decision making at the end of life: shifting sands. J R Soc Med. 2005; 98(7): 297-298.

25. Glare P, Virik K, Jones M, et al. A systematic review of physicians' survival predictions in terminally ill cancer patients. BMJ 2003; 327(7408): 195-198.

26. Fins JJ, Maltby BS, Friedmann E, et al. Contracts, covenants and advance care planning: an empirical study of the moral obligations of patient and proxy. J Pain Symptom Manage 2005; 29(1): 55-68.

27. Jones I, Kirby A, Ormiston P, et al. The needs of patients dying of chronic obstructive pulmonary disease in the community. Fam Pract 2004; 21(3) 310-313.

28. Edmonds P, Karlsen S, Khan S, Addington-Hall J. A comparison of the palliative care needs of patients dying from chronic respiratory diseases and lung cancer. Palliat Med 2001; 15(4): 287-295.

29. Elkington $H$, White P, Addington-Hall J, et al. The healthcare needs of chronic obstructive pulmonary disease patients in the last year of life. Palliat Med 2005; 19(6): 485-491.

30. Solano JP, Gomes B, Higginson IJ. A comparison of symptom prevalence in far advanced cancer, AIDS, heart disease, chronic obstructive pulmonary disease and renal disease. J Pain Symptom Manage 2006; 31(1): $58-69$

31. Seamark DA, Seamark CJ, Halpin DM. Palliative care in chronic obstructive pulmonary disease: a review for clinicians. J R Soc Med 2007; 100(5): 225-233.

32. Jennings AL, Davies AN, Higgins JP, et al. A systematic review of the use of opioids in the management of dyspnoea. Thorax 2002; 57(11): 939-944.

33. Abernethy AP, Currow DC, Frith P, et al. Randomised, double blind, placebo controlled crossover trial of sustained release morphine for the management of refractory dyspnoea. BMJ 2003; 327(7414): 523-528.

34. Taylor SJ, Candy B, Bryar RM, et al. Effectiveness of innovations in nurse led chronic disease management for patients with chronic obstructive pulmonary disease: systematic review of evidence. BMJ 2005 ; 331(7515): 485

35. Thomas K. Caring for the dying at home. Companions on a journey. Oxford: Radcliffe Medical Press, 2003.

DOI: 10.3399/bjgp08X299326

ADDRESS FOR CORRESPONDENCE:

David Halpin,

Royal Devon and Exeter Hospital Department of Respiratory Medicine

Barrack Road, Exeter, EX2 5DW, UK

E-mail: David.Halpin@rdeft.nhs.uk 